\title{
COMMENT
}

\section{EVIMalaR - a model for international cooperation in scientific research}

\author{
Andrew P. Waters \\ The European Virtual Institute for Malaria Research (EVIMalaR) is at risk of losing funding, \\ which could be a major set-back for the European malaria research community.
}

Malaria persists as one of the most deadly infectious diseases in the tropical and subtropical regions of the world. Five species of the Plasmodium parasite are responsible for more than half a million deaths annually, with Plasmodium falciparum being the main species involved. Together, P. falciparum and the more widespread Plasmodium vivax cause an estimated 300 million infections annually, and two-thirds of the global human population is at risk of infection. The resultant economic impact is rarely appreciated, but the 2004 Copenhagen Consensus placed finding a cure for malaria as one of the top four factors that could have a positive impact on the global economy.

But how close are we to finding a cure? Considerable research is devoted to the development of new antimalarial drugs and vaccines, but the evidence to date indicates that the parasite is more than capable of defeating such challenges. Indeed, the emergence of $P$. falciparum resistance to our current front-line drug, artemisinin, in Southeast Asia is the latest set-back ${ }^{1}$, and scientists are now engaged in the struggle to elucidate the molecular basis of this resistance ${ }^{2,3}$. Furthermore, there is only one suboptimal vaccine currently available (RTS,S), and it is likely that resistance to this and other vaccine formulations will also emerge.

In an effort to counteract resistance, there has been substantial investment in the development of novel drugs and vaccines both at governmental level and from nongovernmental organizations, such as the Bill and Melinda Gates Foundation and the Medicines for Malaria Venture (MMV), the latter being the front-runner in the development of new antimalarials. The major pharmaceutical companies have also entered the fray and are contributing key research resources, such as the large chemical libraries of candidate drugs from GlaxoSmithKline. These are laudable and very necessary initiatives, but the rate of drug and vaccine development is slow, and the costs involved remain huge. Moreover, most of these campaigns largely focus on the final development and delivery of lead compounds, which are often identified in academic settings on limited research budgets. Indeed, there is a growing concern in the academic community that the funding emphasis is placed on translational research, without gaining a full understanding of the basic biology of the parasites and their interactions with the human host and mosquito vector. However, the tools to gain such crucial insights are available, and if we are serious about finding a cure for malaria, we have to strategically fund all elements of the cure development pipeline, from basic research through to the discovery of lead compounds and their testing and application. Unfortunately, the discovery phase is the major obstacle; our lack of knowledge of the basic biology of the parasite and of host-parasite interactions means that the transition from lead compounds to therapeutics is still largely serendipitous rather than rational. Therefore, it could be argued that fundamental research should receive a larger proportion of funding in order to maintain the therapeutics pipeline. So, in the absence of adequate quantities of research money, what can be done in a European setting to maximize the efficiency of fundamental malaria research?

The BioMalPar (Biology of Malaria Parasites) initiative was established as a Network of Excellence (NoE) in September 2004 as part of the Sixth Framework Programme (FP6) of the European Commission (EC). One of several NoEs created at the time, BioMalPar was widely perceived as a successful initiative and demonstrated that self-organized communities could harmonize their working practices and increase international collaborations, which were the original goals of the NoEs. Such was the success of BioMalPar that the EC decided to fund a second NoE in 2009 as part of the FP7 programme. Termed the European Virtual Institute for Malaria Research (EVIMalaR), the current network consists of 54 principal investigators from 37 institutes, representing the leading malaria research groups from the European Union. The network also includes six leading African institutions and a prominent institute in India, and also works closely with the Australia-Europe Malaria Research Cooperation (OzEMalaR). A further 20 European research groups are affiliated with EVIMalaR.

The goal of EVIMalaR is almost identical to that of BioMalPar, which was to increase the efficiency of basic malaria research across Europe. This is being achieved through a number of interventions that are coordinated 
by a management infrastructure based at the University of Glasgow, UK. One of the main achievements has been the establishment of the EVIMalaR Ph.D. School, which currently has 23 international students enrolled, following on from the 22 graduates of the BioMalPar School. The school is headed by a dean (Professor Michael Lanzer) at the University of Heidelberg, Germany, and graduate students pursue joint projects in two partner laboratories in different countries, and are affiliated with the European Molecular Biology Organisation. This structuring of the $\mathrm{Ph} . \mathrm{D}$. programme has facilitated the creation of multidisciplinary, integrated research programmes that cover the whole spectrum of malaria research interests, from basic research on the parasite and its modes of transmission, to the clinical investigation of pathogenesis. Looking further ahead, we have initiated additional fostering of the next generation of malaria researchers through the establishment of an EVIMalaR fellowship programme (which currently funds five fellows).

Furthermore, EVIMalaR has enhanced and harmonized experimental approaches through the use of shared technological platforms and exchange visits, and the creation of shared resources (such as EVIMalaR-SEEK and RMgmDB), standard protocols for data collection and reagent banks. These resources and technological platforms are also available online to interested parties from outside the network. Collaboration has not been restricted to the European and African partners: an integrated work plan with OzEMalaR has also been instigated and includes exchange visits, further facilitating global harmonization of research and, we expect, joint publications. As a result of building, expanding on and diversifying its successful history of collaborative research, EVIMalaR has produced 172 peer-reviewed publications in the first 3 years, and some of these articles describe crucial advances in our knowledge of malaria. We are also seeking to establish further network agreements to create and foster global research exchange programmes and to shape future research agendas. Outreach efforts and the communication of EVIMalaR activities have been a priority, and to maximize these goals, the network established a globally recognized annual international meeting (BioMalPar) for basic malaria research, which is a thriving success. The organization also engages with the European vaccine and drug discovery initiatives to ensure that research leads are effectively translated. Furthermore, EVIMalaR has developed outreach programmes and tools for the education of the general public (such as the EVIMalaR comic), and disseminates information on the research and activities of the network to peer groups around the world.

The work of EVIMalaR has had a considerable impact on the coordination of European malaria research and has also led to the creation of new areas of investigation within the field. Through our existing and emerging global contacts, the benefits of our highly coordinated, collaborative approach are becoming apparent worldwide. EVIMalaR is producing a generation of confident young researchers whose modus operandi is research without borders. The Ph.D. school has undoubtedly been the single most effective mechanism for driving the spirit of greater collaboration throughout the network.
Although it is clear that EVIMalaR and its predecessor, BioMalPar, have reshaped the European malaria research landscape, we are now operating on a reduced annual budget of $€ 2.4$ million that is guaranteed only until September 2014. At the moment, it seems unlikely that the EC will offer to fund a continuation of this network, as the NoE initiative will be dropped from the research portfolio. Indeed, a provision of the original agreement was that EVIMalaR should attempt to become self-sustaining. Accordingly, we are currently evolving EVIMalaR into a representative legal entity that will assume certain management and administrative functions, the goal being to raise revenue on behalf of the network members in order to fund long-term programmes of integrated research and training. Although this would enable EVIMalaR to partner members on applications and provide managerial support, there is currently no funding stream that will allow the organization to continue to shape the research agenda. Therefore, a piecemeal portfolio of research funding (for example, funding of the annual conference or Ph.D. school) must be generated, which requires considerable effort from the individual principal investigators, who are already overburdened. One of the benefits of the $\mathrm{NoE}$ format was that it allowed us to organize ourselves, and without funding this might no longer be possible. Our members are involved in individual lobbying of their national governments and private benefactors, as it would take only ten donors to agree to provide the fairly small sum of $€ 1.25$ million ( $€ 250,000$ per partner per annum) to allow the network to continue for another 5 years.

Expansion of the network with appropriate funding could continue to drive collaborative research, not only across Europe but also in other regions of the world. We believe there are strong arguments, as evidenced above, that EVIMalaR is a force for good practice in the international malaria community. With continued funding, EVIMalaR is a model organization for how research can be conducted to achieve greater output and minimize unnecessary duplication of research, not just in the area of malaria research but in any area of biological research. Without support, this 10-year experiment will simply disappear, and all the tangible benefits and achievements will fade away.

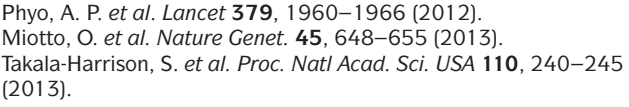

Acknowledgements

The author thanks A. Craig for critical reading of the manuscript. EVIMalaR is funded by the FP7 programme of the EC.

Competing financial interests

The author declares no competing financial interests.

\section{FURTHER INFORMATION}

BioMalPar: http://www.biomalpar.org

Copenhagen Consensus: http://www.copenhagenconsensus.com/projects/

copenhagen-consensus-2004/outcome

EVIMalaR: http://www.EVIMalaR.org

EVIMalaR comic: http://www.malariacomic.com

EVIMalaR Ph.D. School:

http://www.evimalar.org/phd-school/evimalar-phd-school.html

EVIMalaR-SEEK: https://cisbic.bioinformatics.ic.ac.uk/evimalar-seek

RMgmDB: http://www.pberghei.eu/index.php

ALL LINKS ARE ACTIVE IN THE ONLINE PDF 